\title{
Computer-aided Input System for Pairwise Comparison
}

\author{
Zhen Liu, Hikaru Inooka and Masana Kato \\ Graduate School of Engineering, Tohoku University, Aramaki Aza Aoba 01, Scndai 980-8579, Japan \\ Tel. +81-22-217-6961, Fax. +81-22-217-7027, email: liu@elm.mech.tohoku.ac.jp
}

\section{Introduction}

A fundamental aspect of the Analytic Hierarchy Process (AHP, T.L. Saaty) [1] is the use of pairwise comparisons to form positive reciprocal matrices, called pairwise comparison matrix (PCM), its right principal eigenvectors are used as accurate summaries of the decision maker's (DM) judgments. The contradictions which may arise in pairwise comparisons for DMs bring about the inconsistency of the PCM. If the contradictions are to a great extent, then the DM's judgments can not be used for decision making. Therefore, it is expected to develop a method for improving the consistency of PCM. The AHP method is also unsuitbale when a wide-ranging investigation is conducted due to the large volume of data for processing.

In this paper, we propose a computer-aided input system for pairwise comparison (CAISPC) which aids DMs to modify their judgments dynamically in order to improve consistency. It also simplifies data processing.

\section{PCM and Its Consistency}

The PCM $A, A=\left\{a_{i j}\right\}$, is interpreted as follows: every element shows the relative contribution of the $i$ th factor as compared to the $j$ th factor, i.e, $a_{i j}=w_{i} / w_{j}, \quad 1 \leq i \leq n, 1 \leq j \leq n$, where $w_{i}$, $i=1,2, \ldots, n$, represents the weight of factor $i$. The PCM has characteristics of $a_{i i}=1$ and $a_{i j}=\frac{1}{a_{j i}}$, $i, j=1,2, \cdots, n$. Saaty has developed the consistency index (CI) as a measure of the consistency of pairwise comparison matrix.

$$
C I=\frac{\lambda_{\max }-n}{n-1}
$$

where, $\lambda$ is the principal eigenvalue of $A$.

The assumption is that the greater the departure from consistency, the more randomly chosen the DM's entries are in the pairwise comparison matrix. By simulating random reciprocal matrices of different orders, the average consistency indices, known as the random index (RI), can be obtained. The ratio of the CI to the $\mathrm{RI}$ for the same order matrix is called the consistency ratio (CR).

$$
C R=\frac{C I}{R I}
$$

A consistency ratio of 0.10 or less is considered acceptable [2] [3].

If $\lambda=n, C I=0$, then the DM's judgment is called total consistency. In this case, the following equation must be satisfied.

$$
a_{i j}=\frac{a_{k j}}{a_{k i}}
$$

where $k<i$, and $i, j \leq n$. 


\section{The CAISPC System}

The principles of the CAISPC are as following:

- The $n$th order PCM is consistent, when and only when its $(n-1)$ th order sub-matrix is consistent

- To ensure the $n$th order PCM is consistent, the following equation must be satisfied:

$$
\left|a_{i j}-\frac{a_{k j}}{a_{k i}}\right|<\epsilon
$$

where $k<i$, and $i, j \leq n, \epsilon$ is a small enough positive decimal.

- First judgment first priority.

The CAISPC is composed of five parts:

(1) dialogue;

(2) deduction;

(3) data management;

(4) dictionary;

(5) rule base.

Part (1) provides a flexible interface between DM and the system. The DMI can input his or her judgments conveniently with it,' and also can get the feedback from the system whether the judgments made before are consistent or not. According to the feedback DM can modify the judgments with the dialogue interface. People generally find it difficult to recall their previous input, especially with a large criteria. Feedback can give the DM the necessary information for doing further clecisions or modifying previous judgments. Part (2) deducts the consistency of every sub-matrix of the PCM constructed from DM's input according to given rules. Results return to the DM via the dialogue interface. In part (3), raw data, constucted PCM, CI, CR, maximum eigenvalue of the PCM and the corresponding eigenvector are stored in the file system. The file can later be accessed to do further analysis such as cluster analysis. The content of part (4) includes average CI and some other coefficients. Part (5) is made up of consistency deduction rules.

\section{Discussion}

The consistency rate of investigation results can be improved by CAISPC system. As the PCM is constructed automatically according to DM's judgment in the system, data processing can also be simplified.

\section{References}

[1] T.L. Saaty, Axiomatic Foundation of the Analytic Hierarchy Process, Management Science, Vol.32, No.7, 841-855, July 1986.

[2] W.C. Wedley, Consistency Prediction for Incomplete AHP Matrices, Mathematical Computer Modelling, Vol.17, No.4/5, 151-161, 1993.

[3] J.S. Finan and W.J. Hurley, The Analytic Hierarchy Process: Does Adjusting a Pairwise Comparison Matrix to Improve the Consistency Ratio Help?, Computers Operations Research, Vol.24, No.8, 749-755, 1997. 